\section{KURZ GEMELDET}

Allergy News

_ uropas Kinder knabbern zu viel salz-

haltige Snacks und verbringen zu viel Zeit mit Fernsehen oder Computerspielen. Ob damit auch ein erhöhtes Asthmarisiko einhergeht, wollten griechische Wissenschaftler wissen. Sie untersuchten 700 Kinder aus Athen zwischen zehn und zwölf Jahren. Das Risiko, Asthmasymptome zu entwickeln, stieg um den Faktor 4,8 an, wenn die Kinder mehr als dreimal pro Woche Chips oder anderes Salzgebäck knabberten. Diese Assoziation war noch deutlicher bei Kindern, die mehr als zwei

\section{Knabberzeug erhöht Asthmarisiko}

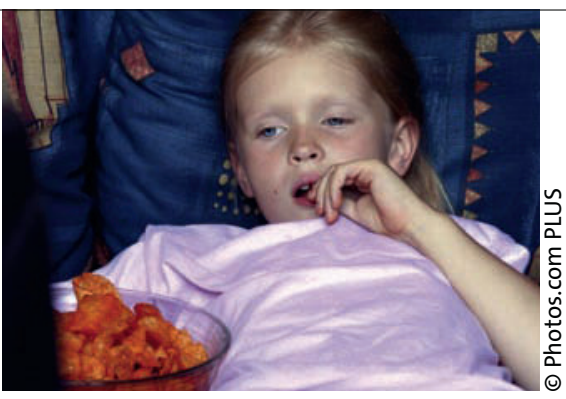

Stunden am Tag vor dem Fernseher oder Computer verbrachten. Weniger häufig von Asthma betroffen waren dagegen Kinder, bei denen typisch mediterrane Ernährung auf dem Programm stand. Frisches Gemüse, Früchte, Fisch und Co. sowie der hohe Anteil an Antioxidanzien taten auch hier ihre Wirkung. Nicola Zink

Arvaniti F et al. J Am Diet Assoc 2011; 111: $251-7$

\section{Ausnahmsweise von der Zuzahlung befreit}

든 in Patient, der unter Bronchialasthma leidet, wird seit 2005 mit einem den Wirkstoff Ciclesonid enthaltenden Medikament behandelt. Da für dieses ein Festbetrag gilt, der weit unter dem Apothekenverkaufspreis liegt, musste der Patient ständig „,draufzahlen“. Schließlich zog er vor Gericht, um die vollständige Kostenübernahme durchzusetzen. Das Sozialgericht Aachen gab ihm Recht. Denn das Asthmamedikament auf Ciclesonid-Basis sei das einzige, das der Patient vertrage. Alle Asthmamittel, die zum Festbetrag erhältlich seien, enthielten andere Wirkstoffe, die bei ihm schmerzhafte Nebenwirkungen auslösten. Das hätten zahlreiche Versuche des Patienten und seines Arztes mit alternativen Präparaten gezeigt. Da der Versicherte also nur mit diesem Mittel ordnungsgemäß behandelt werden könne, müsse die gesetzliche Krankenkasse ausnahmsweise die Kosten in voller Höhe erstatten. Die Zuzahlung sei auf Dauer für den Versicherten unzumutbar. Otto Gritschneder

Sozialgericht Aachen vom 16. November 2010 - S 13 KR 170/10

\section{Doppelter Nutzen}

äuglinge, die hypoallergene Babynahrung erhalten - in der Kuhmilchproteine weitgehend hydrolysiert sind könnten doppelt profitieren. Sie sollen nicht nicht nur weniger anfällig gegenüber Nahrungsmittelallergien sein, sondern auch gegenüber Typ-I-Diabetes. In einer finnischen Studie waren Säuglinge mit familiärem Diabetesrisiko nach dem Abstillen mindestens zwei Monate lang mit hypoallergener Nahrung gefüttert worden. Sie entwickelten bis zum Alter von zehn Jahren nur halb so oft mindestens einen bzw. zwei diabetestypische Autoantikörper wie Säuglinge, die Kuhmilch bekommen hatten (17 vs. $30 \%$ bzw. 7 vs. 16\%). Die Untersuchung mit 230 Kindern ist Teil einer laufenden größeren Studie, die endgültig Aufschluss über den Nutzen dieser Intervention bringen soll.

Dirk Einecke

Knip M et al. N Engl J Med 2010; 63:1900-8

\section{Zu teuer bezahlter Chic}

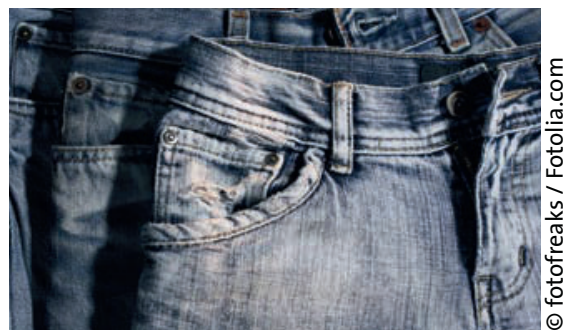

\section{Kann Mann damit hyposensibilisieren?}

B ei manchen Männern treten nach dem Orgasmus Kopfschmerzen und grippeähnliche Symptome wie Augenbrennen, eine laufende Nase und Fieber auf, die stunden- oder tagelang anhalten können. Marcel Waldinger und sein Team von der Universität Utrecht befassten sich

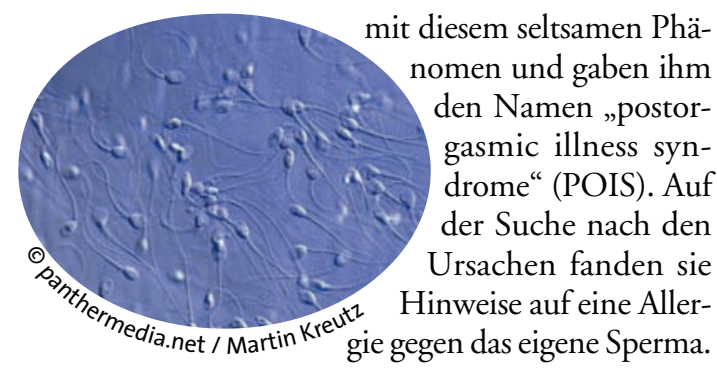

Von 45 POIS-Patienten fühlte sich keiner krank, wenn er masturbierte, ohne zum Samenerguss zu kommen. Sobald die Männer aber ejakulierten, traten die Symptome auf. Nach einem Pricktest mit der eigenen Samenflüssigkeit zeigten sich bei 29 von 33 Männern Rötungen. Zwei der Betroffenen unterzogen sich einer subkutanen Hyposensibilisierung mit einer verdünnten Lösung ihrer eigenen Samenflüssigkeit. Bei einem besserten sich die Symptome nach einem Jahr, beim anderen erst nach drei Jahren.

Josef Mehrl

Waldinger MD et al. J Sex Med 2011 Jan 17 [Epub ahead of print]
_ ine gängige Praxis, um neue Jeans - gebraucht aussehen zu lassen, ist die Bearbeitung mit einem Sandstrahler. Das birgt erhebliche Gefahren für die Textilarbeiter. Ein Pneumologenteam um Nur Dilek Bakan aus Istanbul, Türkei, dokumentierte die Daten von 32 jungen Männern, die in den Jahren 2001 bis 2009 aufgrund Jeans-Sandstrahlarbeiten an einer Silikose erkrankt waren. Sechs (19\%) der Patienten verstarben, von den übrigen entwickelten 16 (62\%) eine fortschreitende schwere Lungenfibrose. Die durchschnittliche Überlebenszeit betrug nur 78 Monate, die geschätzte 5-Jahres-Überlebensrate liegt bei 69\%. Ute Kempf

European Respiratory Society Januar 2011 